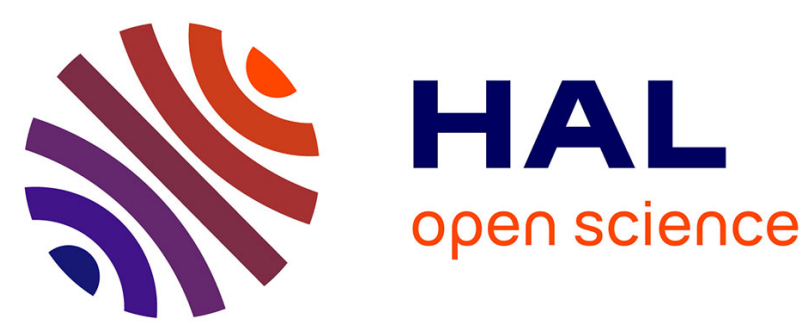

\title{
MESURE DE VITESSE PAR DOPPLER \\ ULTRASONORE : COMPARAISON DE DIFFÉRENTES TECHNIQUES D'ANALYSE TEMPS-FRÉQUENCE
}

C. Cachard, Y. Zhu, G. Gimenez, F. Peyrin

\section{To cite this version:}

C. Cachard, Y. Zhu, G. Gimenez, F. Peyrin. MESURE DE VITESSE PAR DOPPLER ULTRASONORE: COMPARAISON DE DIFFÉRENTES TECHNIQUES D'ANALYSE TEMPS-FRÉQUENCE. Journal de Physique Colloques, 1990, 51 (C2), pp.C2-663-C2-666.

10.1051/jphyscol:19902154 . jpa-00230456

\section{HAL Id: jpa-00230456 https://hal.science/jpa-00230456}

Submitted on 1 Jan 1990

HAL is a multi-disciplinary open access archive for the deposit and dissemination of scientific research documents, whether they are published or not. The documents may come from teaching and research institutions in France or abroad, or from public or private research centers.
L'archive ouverte pluridisciplinaire HAL, est destinée au dépôt et à la diffusion de documents scientifiques de niveau recherche, publiés ou non, émanant des établissements d'enseignement et de recherche français ou étrangers, des laboratoires publics ou privés. 
COLLOQUE DE PHYSIQUE

Colloque C2, supplément au $n^{\circ} 2$, Tome 51, Février 1990

ler Congrès Français d'Acoustique 1990

MESURE DE VITESSE PAR DORPLER ULTRASONORE : COMPARAISON DE DIFFERENTES TECHNIQUES D'ANALYSE TEMPS-FREQUENCE

\author{
C. CACHARD, Y.M. ZHU, G. GIMENEZ et F. PEYRIN \\ Laboratoire "Traitement du Signal et Ultrasons". INSA Bât. 502, 20 \\ Avenue Albert Einstein, F-69621 Villeurbanne Cedex, France
}

Résumé : L'effet Doppler permet de transposer une mesure de vitesse en une mesure de fréquence. Lorsque la vitesse d'une cible évolue en fonction du temps une analyse spectrale du signal Doppler est insuffisante. Dans cette article nous comparons, sur des signaux de simulation et sur des signaux réels de bulles en mouvement, les performances de deux techniques fournissant la fréquence instantanée d'un signal : une méthode basée sur le calcul du signal analytique associé au signal Doppler et la Transformation de Wigner-Ville.

\begin{abstract}
The Doppler effect allows to transpose a velocity measurement in a frequency measurement. If the velocity is time-dependent, a spectrum analyse of the Doppler signal is inadequate. In this paper we compare, from simulated signals and from Doppler signal of travelling bubbles, the accuracy of two signal processing methods to obtain the instantaneous frequency : the first one computes the analytic signal associated to the Doppler signal while the second one uses the Wigner-Ville Transform.
\end{abstract}

\title{
1 - INTRODUCTION
}

La vélocimétrie ultrasonore basée sur l'effet Doppler est une technique très utilisée pour mesurer soit la vitesse d'écoulement d'un fluide soit la vitesse d'une cible dans un milieu au repos. Dans cette technique la vitesse de la cible est proportionnelle à l'écart existant entre la fréquence du signal émis et la fréquence du signal reçu. Cet écart de fréquence est appelé fréquence Doppler. La mesure d'une vitesse est donc transposée en la mesure d'une fréquence. Un dispositif de mesure est constitué d'un transducteur émetteur et d'un traducteur récepteur dont l'intersection des faisceaux ultrasonores définit un volume dans lequel s'effectue la mesure de vitesse. Le signal à la fréquence Doppler $\mathrm{fD}=\omega_{\mathrm{D}} / 2 \pi$, appelé signal Doppler, est obtenu par démodulation analogique du signal issu du traducteur récepteur. Une analyse spectrale de ce signal fournit la fréquence Doppler si le signal est monochromatique c'est à dire si la vitesse de la cible est constante. Si par contre, cette vitesse évolue en module ou/et en direction en fonction du temps le spectre du signal Doppler se présente sous forme d'un pic plus ou moins large, et seule une valeur moyenne de la fréquence, et donc de la vitesse peut-être déduite. II faut alors faire appel à la notion de fréquence instantanée.

Dans cet article, après une brève description du principe du vélocimètre ultrasonore, nous considérons les méthodes de traitement du signal permettant de suivre l'évolution du contenu fréquentiel du signal Doppler. Plus précisément, nous nous intéressons à l'estimation de la fréquence instantanée à partir du calcul du signal analytique associé au signal Doppler et de la Transformation de Wigner-Ville (TWV). La première technique est basée sur le calcul de la transformation de Hilbert. Le résultat obtenu se présente sous forme d'une courbe temps-fréquence. La deuxième technique consiste à transformer le signal Doppler monodimensionnel en une image bidimensionnelle temps-fréquence. La trace des maximum locaux dans ce plan donne alors la fréquence instantanée du signal. A partir de signaux simulés, nouś avons comparé les avantages et les limites de chacune de ces méthodes et nous avons évalué leurs performances dans des situations correspondant à divers comportements d'une cible dans le volume de mesure. Enfin, les résultats de ces méthodes appliquées à des signaux réels (signaux réfléchis par des bulles en mouvement) sont présentés.

\section{2 - VELOCIMETRE ULTRASONORE}

Le principe du vélocimètre est représenté sur la figure 1 et a été exposé dans $[1,2]$. Soit ue(t) un signal sinusoïdal à la pulsation we qui attaque le traducteur ultrasonore. Celui-ci émet une onde ultrasonore qui rencontre la cible se déplaçant à la vitesse $v$. L'onde ultrasonore rayonnée par cette cible en mouvement par rapport aux traducteurs ultrasonores est à la pulsation $\omega_{r}(t)=\omega_{e}+\omega_{D}(t): c$ 'est l'effet Doppler. Le traducteur récepteur capte une partie de cette onde et délivre un signal $u_{r}(t)$ à la pulsation $\omega_{r}(t)$. Après amplification, ce signal est démodulé par un amplificateur synchrone qui délivre deux signaux en quadrature à la pulsation Doppler $\omega_{D}(t)$. Ce sont ces signaux qui doivent être traités pour en extraire la fréquence Doppler. 
Fig. 1 - Schéma de principe du vélocimètre ultrasonore

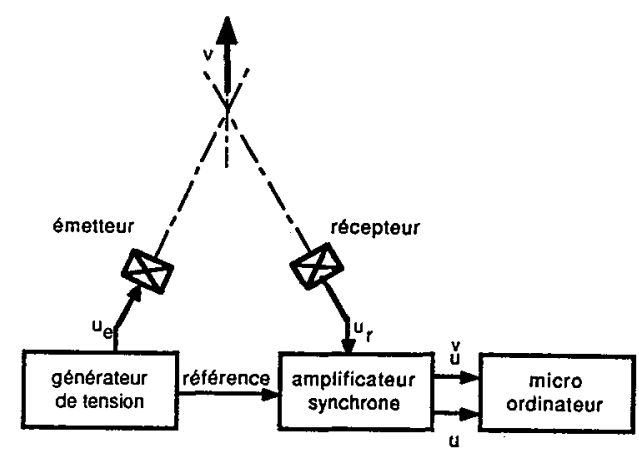

\section{3 - EVALUATION DE LA FBEQUENCE INSTANTANEE D'UN SIGNAL}

\section{1 - Le signal analytique}

Le signal analytique $\mathrm{u}(\mathrm{t})$ est un signal complexe associé au signal physique $\mathrm{u}(\mathrm{t})$ et qui possède certaines propriétés [3]. Il est défini par:

$$
\underline{u}(t)=u(t)+j u(t)
$$

où $\stackrel{v}{u}(t)$ est la transformée de Hilbert de $u(t), u(t)$ et $\stackrel{v}{u}(t)$ sont alors en quadrature. L'écriture sous forme polaire du signal analytique permet de définir l'enveloppe réelle $r(t)$ par le module du signal analytique et la phase instantanée $\Phi(t)$ par son argument:

$$
r(t)=|\underline{\underline{u}}(t)|=\left[u^{2}(t)+u^{2}(t)\right]^{1 / 2} \quad \text { et } \quad \Phi(t)=\arg [u(t)]=\arctan [u(t) / u(t)]
$$

La dérivée temporelle de cette phase instantanée représente la pulsation instantanée $\mathbf{z}_{\mathbf{i}}(\mathbf{t})$ du signal physique $u(t)$

$$
\omega_{i}(t)=2 \pi f_{i}(t)=\frac{d \Phi(t)}{d t}
$$

Les deux signaux en quadrature délivrés par l'amplificateur synchrone peuvent être pris comme la partie réelle $u(t)$ et la partie imaginaire $u(t)$ d'un signal analytique dont la fréquence instantanée est la fréquence Doppler [4].

\section{2 - La Transformation de Wianer-Ville}

La TWV est une méthode pour représenter des signaux non-stationnaires [5]. Elle permet d'accéder, de manière précise, à la fréquence instantanée du signal. Etant donné un signal temporel $u(t)$ (réel ou complexe), sa TWV est définie par :

$$
W_{u}(t, f)=\int_{R} u(t+\theta / 2) u^{*}(t-\theta / 2) \exp (-j 2 \pi f \theta) d \theta
$$

où * représente l'opérateur de conjugaison complexe.

\section{4 - APPLICATIONADES SIGNAUX}

\section{1 - Signaux simulés}

Afin d'apprécier les performances des deux techniques précédentes, nous avons choisi de travailler dans un premier temps sur des signaux simulant différentes configurations rencontrées lors de la mesure de vitesse par effet Doppler. Seul les cas les plus caractéristiques sont présentés ici. Le premier exemple correspond à un signal sinusoïdal de pulsation $\omega_{1}$, modulé en amplitude par une fenêtre de Hanning et modulé linéairement en fréquence (fig.2a). La modulation d'amplitude correspond à une amplitude variable du champ acoustique traversé par la cible (divergence des traducteurs) alors que la modulation de fréquence traduit l'évolution de la vitesse de la cible. 


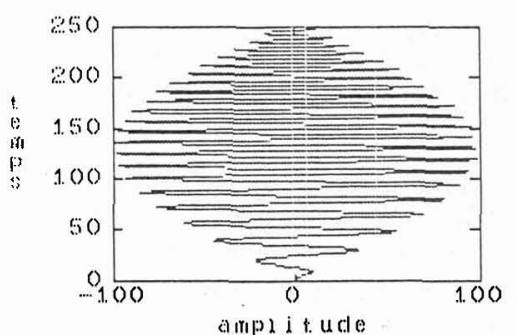

a

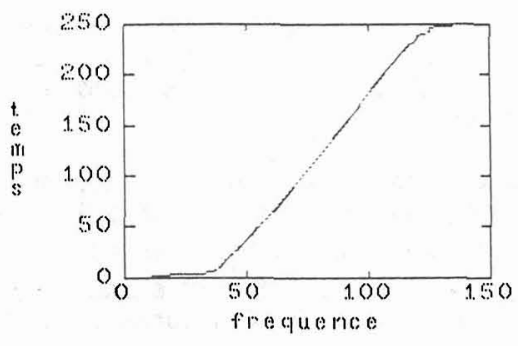

b

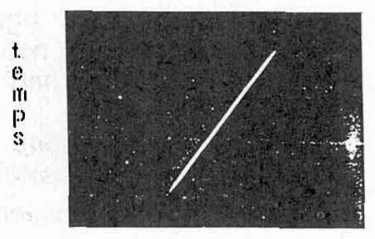

Prequerce

Fig. 2 - a) Signal modulé en fréquence et en amplitude; b) fréquence instantanée calculée à partir du signal analytique ; c) TWV.

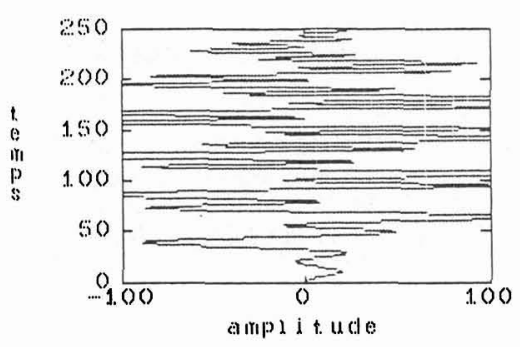

a

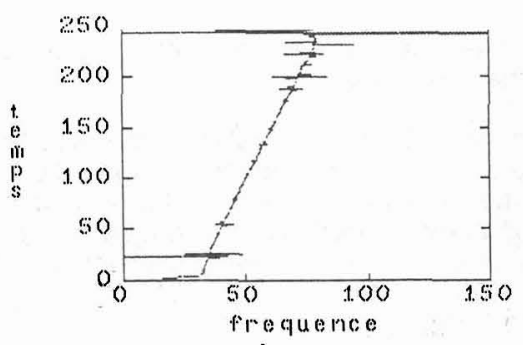

b

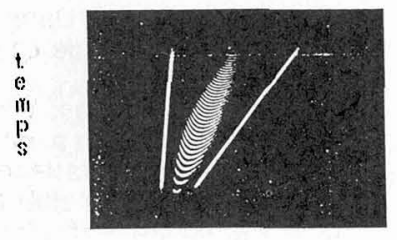

freature

Fig. 3 - a) Signal à deux composantes ; b) fréquence instantanée calculée à partir du signal analytique ; c) TWV.

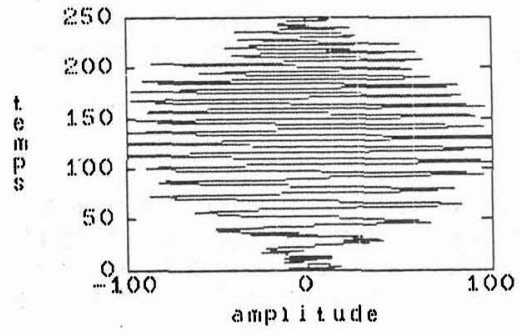

a

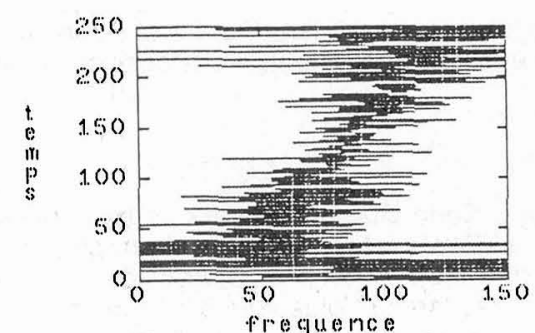

b

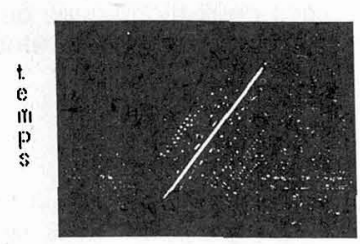

frecture e

Fig. 4 - a) Signal bruité ; b) fréquence instantanée calculée à partir du signal analytique ; c) TWV.

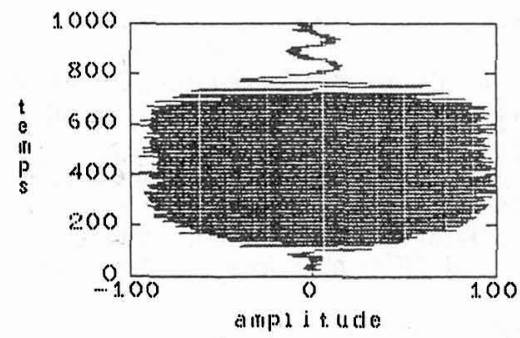

a

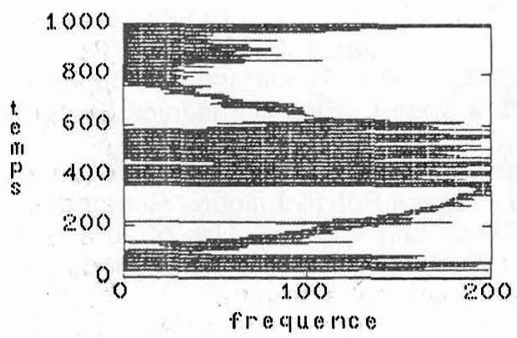

b

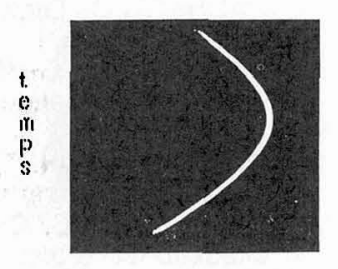

frequere

Fig. 5 - a) Signal Doppler d'une bulle déviée ; b) fréquence instantanée calculée à partir du signal analytique ; c) TWV. 
La fréquence instantanée obtenue en appliquant les relations (2) et (3), respectivement la relation (4) est représentée sur la figure $2 \mathrm{~b}$, respectivement figure $2 \mathrm{c}$. Le signal ci-dessus étant un signal monocomposante, la notion de fréquence instantanée s'applique naturellement. Les résultats fournis par ces deux techniques sont donc équivalents.

Par contre considérons dans un deuxième exemple un signal contenant deux composantes à des fréquences différentes tel que celui représenté sur la figure 3a. II est constitué par la somme du signal précedent et d'un deuxième signal semblable dans lequel la pulsation est $\omega_{2}=\omega 1 / 2$. Cette configuration correspond à deux cibles ayant des vitesses différentes présentes en même temps dans le volume de mesure. La méthode du signal analytique (fig. $3 b$ ) donne pour chaque instant t une seule valeur de fréquence. En utilisant les relations (2) et (3) le calcul analytique de la fréquence instantanée conduit effectivement à :

$$
\omega(t)=\frac{1}{2}\left\{\omega_{1}(t)+\omega_{2}(t)+t \frac{d\left[\omega_{1}(t)+\omega_{2}(t)\right]}{d t}\right\}
$$

Tandis que la TWV (fig. 3c) fournit deux segments distincts, chacun représentant la fréquence instantanée d'une composante. Dans ce cas, seule la TWV permet d'interpréter le phénomène physique, c'est à dire d'obtenir la vitesse de chacune des cibles.

Enfin on s'est intéressé à un signal bruité (fig. 4a) afin de tester la sensibilité au bruit de chacune de ces techniques. Un bruit aléatoire a été ajouté au signal de départ (fig. 2a) tel que le rapport signal sur bruit est de 13,7 dB. Avec la technique du signal analytique (fig. 4b) l'appréciation de la fréquence instantanée s'avère difficile. En effet l'opérateur de dérivation, appliqué à la phase instantanée, est très sensible au bruit. Par contre le résultat obtenu avec la TWV est peu influencé par le bruit (fig. 4c)

\section{2 - Signaux de bulles en mouvement}

La figure 5a représente le signal en phase délivré par le détecteur synchrone du vélocimètre Doppler lorsqu'une seule bulle est présente dans le volume de mesure. Cette expérience correspond à la mesure de la vitesse d'une bulle déviée par un champ ultrasonore intense [6]. Comme le prévoyait l'étude sur des signaux simulés, dans le cas d'un signal monocomposante (une seule bulle), les résultats obtenus avec ces deux techniques de traitement concordent (fig. 5b et 5c) bien que le bruit soit important sur la figure 5b. Le phénomène étudié évoluant lentement, il est possible d'effectuer un filtrage haute fréquence du résultat ainsi obtenu.

\section{5 - CONCLUSION}

Pour la mesure de la vitesse d'une cible unique les deux techniques donnent des résultats équivalents. Signalons toutefois que la TWV est d'une utilisation plus complexe (temps de calcul important, représentation sous forme d'image). La technique du signal analytique sera donc préférée dans le cas de signaux peu bruités. Par contre, lorsque plusieurs cibles sont présentes dans le volume de mesure seule la TWV permet d'obtenir la vitesse de chacune d'entre elles. Le signal analytique ne permet pas d'interpréter le phénomène physique. Notons, que l'utilisation commune de ces deux méthodes peut apporter une aide supplémentaire dans l'interprétation des résultats.

\section{Bibliographie}

1 - CACHARD C., GIMENEZ G., VRAY D. Mesure ultrasonore du vecteur vitesse de bulles en mouvement dans un liquide, Revue d'Acoustique, 1986, 78, p.19-23.

2 - CACHARD C., GIMENEZ G., VRAY D. Ultrasonic Doppler device for measurement of time-dependent and space-dependent flow speed. IEEE Ultrasonics Symposium, 1988, Chicago-USA, vol. 2, p. 901904.

3 - F. DE COULON. Théorie et traitement des signaux (chaps. 7 and 11), vol. VI, Traité d'électricité. Lausanne, Switzerland, Presses Polytechniques Romandes, 1984.

4 - GIMENEZ G., CACHARD C. and VRAY D. Use of an analytic signal to model interaction between an acoustic wave and a moving target with a time-dependent velocity. IEEE transactions on ultrasonics, ferroelectrics and frequency control, à paraître.

5 - FLANDRIN P. Representation temps-fréquence des signaux non-stationnaires", Thèse Doct.-etat INPG, Grenoble, 1987.

6 - CACHARD C., GIMENEZ G., VRAY D. Theoretical and experimental investigation of acoustic radiation pressure acting on a single bubble. 13ème international Congress on Acoustics, 1989, BelgradeYougoslavie, vol. 1, p. 347-350. 